\title{
Collagen VI Is Upregulated in COPD and Serves Both as an Adhesive Target and a Bactericidal Barrier for Moraxella catarrhalis
}

\author{
Suado M. Abdillahi ${ }^{a}$ Marta Bober ${ }^{a}$ Sara Nordin ${ }^{a}$ Oskar Hallgren ${ }^{b}$ \\ Maria Baumgarten $^{a}$ Jonas Erjefält ${ }^{c}$ Gunilla Westergren-Thorsson ${ }^{d}$ \\ Leif Bjermer $^{b} \quad$ Kristian Riesbeck $^{\mathrm{e}} \quad$ Arne Egesten $^{\mathrm{b}}$ Matthias Mörgelin ${ }^{\mathrm{a}}$
}

Divisions of a Infection Medicine and bespiratory Medicine and Allergology, Department of Clinical Sciences, and Divisions of ${ }^{\mathrm{C}}$ Airway Inflammation and Immunology and ${ }^{\mathrm{d}}$ Lung Biology, Department of Experimental Medical Science, Lund University, Lund, and 'Division of Medical Microbiology, Department of Laboratory Medicine Malmö, Lund University, Malmö, Sweden

\section{Key Words}

Adhesion · Bronchopulmonary infection - Collagen VI .

Chronic obstructive pulmonary disease $\cdot$ Extracellular matrix · Innate immunity · Moraxella catarrhalis · Pulmonary fibrosis

\footnotetext{
Abstract

Moraxella catarrhalis is a Gram-negative human mucosal commensal and pathogen. It is a common cause of exacerbation in chronic obstructive pulmonary disease (COPD). During the process of infection, host colonization correlates with recognition of host molecular patterns. Importantly, in COPD patients with compromised epithelial integrity the underlying extracellular matrix is exposed and provides potential adhesive targets. Collagen VI is a ubiquitous fibrillar component in the airway mucosa and has been attributed both adhesive and killing properties against Gram-positive bacteria. However, less is known regarding Gram-negative microorganisms. Therefore, in the present study, the interaction of M. catarrhalis with collagen VI was characterized. We found that collagen $\mathrm{VI}$ is upregulated in the airways of COPD patients and exposed upon epithelial desquamation. Ex
}

vivo, we inoculated airway biopsies and fibroblasts from COPD patients with $M$. catarrhalis. The bacteria specifically adhered to collagen VI-containing matrix fibrils. In vitro, purified collagen VI microfibrils bound to bacterial surface structures. The primary adhesion target was mapped to the collagen $\mathrm{VI} \mathrm{a}_{2}$-chain. Upon exposure to collagen $\mathrm{VI}$, bacteria were killed by membrane destabilization in physiological conditions. These previously unknown properties of collagen $\mathrm{VI}$ provide novel insights into the extracellular matrix innate immunity by quickly entrapping and killing pathogen intruders.

(c) 2015 S. Karger AG, Basel

\section{Introduction}

Moraxella catarrhalis is a nonencapsulated aerobic diplococcus and a well-adapted commensal of the human respiratory tract. During the past decades, it has gained increasing attention as a mucosal pathogen [1-3] with the

S.M. Abdillahi and M. Bober contributed equally to this investigation.

\section{KARGER 125}

(c) 2015 S. Karger AG, Basel

$1662-811 \mathrm{X} / 15 / 0075-0506 \$ 39.50 / 0$

E-Mail karger@karger.com

www.karger.com/jin
Dr. Matthias Mörgelin

Department of Clinical Sciences, Division of Infection Medicine, Lund University Biomedical Center, Floor B14

SE-22184 Lund (Sweden)

E-Mail matthias.morgelin@ med.lu.se 
potential to cause upper and lower respiratory tract infections. In preschool children $M$. catarrhalis is the third most common cause of infective otitis media [4]. In adults this pathogen mainly causes sinusitis, laryngitis, bronchitis and pneumonia [5]. Most commonly, however, $M$. catarrhalis is associated with persistent bacterial colonization in chronic obstructive pulmonary disease (COPD) patients. It is responsible for $10-15 \%$ of infective COPD exacerbations $[5,6]$ and, taken together, represents a significant global health challenge.

The integrity of body barriers is crucial for the protection of the host against invasive pathogens and transmitted diseases. In order to cause invasive infection, pathogens breach these barriers by exploiting ports of entry and subsequently establish an infection within the surrounding tissue. An initial step in this carefully orchestrated series of events is host target recognition, followed by primary adhesion. By this means bacterial engagement of specific host tissue structures may direct a pathogen to a particular niche, establishing persistent infection and inducing invasion. As a consequence, bacteria have evolved adhesins with the capacity to mediate a highly specific recognition of host molecular patterns. In addition to epithelial cell surface receptors, evidence is emerging that connective tissues provide adhesive sites for pathogens. In COPD patients with predisposing conditions such as chronic inflammation, viral infections or mechanical damage, the epithelial cell layer integrity is compromised and the underlying connective tissue becomes exposed. In this situation, a spectrum of extracellular matrix targets is available for recognition by bacterial adhesins. In recent work, fibronectin, laminin, vitronectin, and collagens I and IV have been identified as such targets [7-13].

Collagen VI is a large multidomain molecule and a ubiquitous fibrillar extracellular matrix constituent of connective tissues distant to basement membranes [14, 15]. In some tissues it has been reported as a basement membrane component [16]. It is a heterotrimer of different $\alpha$-chains, which are organized as a short central triple helix, flanked by various globular domains sharing homology with von Willebrand factor type A domain, salivary gland proteins, fibronectin type III repeat, and bovine pancreatic trypsin inhibitor/Kunitz domain [17-19]. In a previous study, collagen VI was shown to be upregulated in the airway walls during tissue remodeling in asthma [20]. Recently we identified this collagen as an adhesive substrate for the Gram-positive pathogens Streptococcus pyogenes and Streptococcus pneumoniae [21]. In addition, we found that collagen VI kills streptococci, implying a novel role in connective tissue innate immunity by encoding both adhesive and antimicrobial properties [22].

During our studies we noticed that Gram-negative respiratory pathogens also adhere to this collagen, followed by bacterial killing, and were prompted to dissect the underlying molecular mechanisms in further detail. As a first step, we aimed to unravel the role of the different collagen VI domains in primary adhesion and chose $M$. catarrhalis as a model. In the present study we provide evidence that collagen VI is significantly upregulated in the airways of COPD patients. In patches of epithelial damage, extracellular collagen VI networks are exposed in the airway mucosa distant to basement membranes and accessible to intruding bacterial pathogens. In this environment, collagen VI is a specific substrate for $M$. catarrhalis adherence, followed by membrane destabilization and killing at physiological $\mathrm{pH}$ and ionic strength. In particular, the collagen VI $\alpha_{2}$-chain was identified as the primary adhesion target. This is the first report of such properties of collagen VI and implies important dual properties of this molecule in bacterial pathogenesis and innate host defense.

\section{Materials and Methods}

\section{Bacterial Strains and Growth Conditions}

Moraxella catarrhalis was grown on chocolate agar plates or in brain-heart infusion liquid broth (Difco, Detroit, Mich., USA). Nontypeable Haemophilus influenzae were cultured on chocolate agar plates or in brain-heart infusion liquid broth supplemented with nicotinamide adenine dinucleotide and hemin (both at $10 \mu \mathrm{g}$ / $\mathrm{ml})$. Streptococcus pneumoniae was cultured on blood agar plates or in Todd-Hewitt broth supplemented with $0.5 \%$ yeast extract (Difco). Bacteria were grown to $2 \times 10^{9} \mathrm{CFU} / \mathrm{ml}$ at $37^{\circ} \mathrm{C}$ in a humid atmosphere containing $5 \% \mathrm{CO}_{2}$. Finegoldia magna bacteria were cultured under strict anaerobic conditions in Todd-Hewitt broth supplemented with $0.5 \%$ Tween -80 at $37^{\circ} \mathrm{C}$.

\section{Human Lung Tissues}

The present study involved 11 subjects with moderate to severe COPD (GOLD stages II-IV). Two additional groups were used as controls: ex-smokers or current smokers without COPD $(n=9)$ and a control group with subjects who had never smoked $(n=10)$. Lung tissue was obtained in association with lung lobectomy due to suspected lung cancer, a routine procedure to collect tissues from COPD patients. Only patients with solid tumors with visible borders were included in the study, and tissue was obtained as far from the tumor as possible. Tissue biopsies from smoking and neversmoking subjects were obtained using the same procedure as for otherwise healthy nonatopic individuals. For histological sample preparation, care was taken to immerse the tissue in $4 \%$ paraformaldehyde immediately after surgical excision. All subjects gave their written informed consent to participate in the study, which was approved by the local ethics committee in Lund, Sweden (LU339-00). 
cDNA Synthesis and Real-Time PCR

To analyze the expression of collagen type VI in lung tissues from healthy, smoking and COPD individuals, total RNA was isolated. Frozen lung tissue samples were transferred to RNA-Later ice (Ambion). Total RNA was purified in 2 steps: first, Trizol (Invitrogen, Calif., USA) followed by RNeasy (Qiagen, Crawley, UK). Reverse transcription was performed using a High-Capacity cDNA Reverse Transcription kit (Applied Biosystems) according to the manufacturer's instructions. Real-time PCR was performed using TaqMan probe-based chemistry (Applied Biosystems). Primers/probes for collagen type VI $a_{2}$-chain (Hs00365167_m1) and the endogenous control glyceraldehyde-3-phosphate dehydrogenase (GAPDH) and $3.2 \mu \mathrm{l}$ cDNA were combined in $25 \mu \mathrm{l}$ total reaction volume with $4 \mu \mathrm{l}$ probe for collagen type VI $\alpha_{2}$-chain or GAPDH. The amplification reactions were performed in triplicates in an ABI Prism 7000 Sequence Detection System (Applied Biosystems) according to the manufacturer's instructions. Data were collected and analyzed using the Sequence Detector v1.1 software (Applied Biosystems). Relative gene expression was calculated using the comparative threshold cycle method according to Livak and Schmittgen [23].

\section{Human Pulmonary Fibroblasts and Cell Culture Conditions}

Fibroblasts were isolated from biopsies from control subjects and from tissue sample explants obtained from COPD patients. After sampling, biopsies were immediately transferred to cell culture medium (DMEM supplemented with 10\% FBS, ascorbate 100 $\mu \mathrm{g} / \mathrm{ml}$, gentamicin, protein tyrosine phosphatase and amphotericin B; all from Gibco BRL, Paisley, UK). After rinsing, biopsies were chopped into small pieces that were allowed to adhere to the plastic of cell culture flasks for $4 \mathrm{~h}$ and kept in cell culture medium at $37^{\circ} \mathrm{C}$ until outgrowth of fibroblasts was observed. Lung explants were obtained from different patient cohorts with severe COPD (GOLD stage IV) who had given informed consent. The study was fully approved by the Swedish Research Ethical Committee in Lund (FEK 91/2006 and FEK 213/2005). Briefly, lung explants were dissected directly after removal from COPD patients, and specimens were immediately transferred to cell culture medium. Bronchial tissue was collected from the luminal side from the same localization as where bronchial biopsies were taken. Bronchial specimens were chopped into small pieces and then treated as the biopsy material described above. Experiments were performed at passages 3-6. The cell cultures were continuously stained with antibodies against vimentin and prolyl-4-hydroxylase to verify the mesenchymal identity and to estimate the purity.

\section{Adhesion of M. catarrhalis to Human Lung Biopsies}

Paraffin-embedded human lung tissue samples from healthy and COPD subjects were sectioned and mounted on glass slides according to routine protocols. For bacterial adhesion experiments, they were deparaffinized with Tissue Clear and rehydrated through a descending series of ethanol (from 100 to $50 \%$ ). The specimens were put into a wet chamber and inoculated with $2 \times$ $10^{9} \mathrm{CFU} / \mathrm{ml}$ of $M$. catarrhalis in PBST (PBS containing $0.05 \%$ Tween-20) for $1 \mathrm{~h}$ at $37^{\circ} \mathrm{C}$. In the antibody-blocking assays, paraffin sections were preincubated for $30 \mathrm{~min}$ at room temperature with $10 \mu \mathrm{g} / \mathrm{ml}$ anti-collagen VI. In the collagen VI-blocking assays $2 \times 10^{9} \mathrm{CFU} / \mathrm{ml}$ bacteria were preincubated for $30 \mathrm{~min}$ at room temperature with $10 \mu \mathrm{g} / \mathrm{ml}$ soluble collagen VI. Unbound bacteria were removed by extensive washing with PBST. Tissue sections were covered with brain-heart infusion medium and incubated in $5 \% \mathrm{CO}_{2}$ at $37^{\circ} \mathrm{C}$ for $5 \mathrm{~h}$ to allow bacterial multiplication. Unbound bacteria were again removed by extensive washing with PBST. After incubation with bacteria, tissue sections were fixed with $2.5 \%$ glutaraldehyde in cacodylate buffer $(0.15 \mathrm{M}$ sodium cacodylate, $\mathrm{pH}$ 7.4) overnight at room temperature. Specimens were prepared for scanning electron microscopy as described below.

\section{Adhesion of M. catarrhalis to Human Pulmonary Fibroblasts}

Fibroblasts from healthy and COPD lung tissues were incubated with $2 \times 10^{9} \mathrm{CFU} / \mathrm{ml}$ of bacteria for $1 \mathrm{~h}$ at $37^{\circ} \mathrm{C}$ in a humid atmosphere with $5 \% \mathrm{CO}_{2}$. In parallel, blocking assays with anticollagen VI or soluble collagen VI were carried out as described in the paragraph above. After a washing step, cells were fixed with $4 \%$ formaldehyde and $2.5 \%$ glutaraldehyde in PBS for $2 \mathrm{~h}$ at room temperature. In some experiments, for pre-embedding immunogold labeling of collagen VI in matrix fibrils, sections were incubated with a 1:100 dilution of anti-collagen VI antibody and protein A/G-coated colloidal gold particles (10 nm; British BioCell International, Cardiff, UK). After washing, samples were fixed with $4 \%$ formaldehyde and $2.5 \%$ glutaraldehyde. Specimens were subsequently prepared for scanning electron microscopy as described below.

\section{Reagents, Labeling of Proteins and Binding Assays}

Collagen type VI microfibrils were extracted from bovine cornea by collagenase digestion [24] with modifications as described in Bober et al. [21]. Antibodies (1014+) and recombinant collagen VI fragments [ $\alpha_{1}(\mathrm{VI}), \alpha_{2}(\mathrm{VI}), \alpha_{3}(\mathrm{VI}), \mathrm{N} 9-\mathrm{N} 2$ and N6-C5) were a kind gift of Dr. Rupert Timpl (MPI, Martinsried, Germany; for references, see Bruns et al. [15] and Timpl and Chu [25]). Proteins were radiolabeled with ${ }^{125}$ I using iodobeads (Pierce, Rockford, Ill., USA) according to the manufacturer's specifications. Binding of radiolabeled protein to bacteria was performed as described [21]. For electron microscopy recombinant collagen VI $\alpha$-chains and fragments were labeled with $5 \mathrm{~nm}$ thiocyanate gold as described earlier [26]. Bacteria were grown to mid-logarithmic phase, washed and diluted to $2 \times 10^{9} \mathrm{CFU} / \mathrm{ml}$ in Tris-buffered saline $(150 \mathrm{mM}$ $\mathrm{NaCl}, 50 \mathrm{~mm}$ Tris-HCl, pH 7.4) supplemented with $5 \mathrm{mM}$ glucose (TG buffer) and then incubated with gold-labeled proteins for $1 \mathrm{~h}$ at $37^{\circ} \mathrm{C}$. Samples were prepared for negative staining as described below.

\section{Light Microscopy and Immunohistochemistry}

For immunostaining, airway biopsies from healthy and COPD patients were fixed in $4 \%$ formaldehyde, dehydrated and embedded in paraffin. Sample sections were placed on Superfrost Plus glass slides (Fisher Scientific, Gothenburg, Sweden), deparaffinized in Tissue Clear (Histolab Products, Gothenburg, Sweden) and rehydrated in graded alcohol and water. The sections were then treated with Dako Antigen Retrieval Solution (Dako, Glostrup, Denmark) for $40 \mathrm{~min}$ at $97^{\circ} \mathrm{C}$. The samples were blocked in Tris-buffered saline ( $150 \mathrm{mM} \mathrm{NaCl}, 50 \mathrm{~mm}$ Tris- $\mathrm{HCl}, \mathrm{pH}$ 7.4) with $0.05 \%$ Tween- 20 supplemented with $20 \%$ goat serum, and $1 \%$ BSA for $45 \mathrm{~min}$ at room temperature. After 3 washing steps, they were incubated overnight at room temperature with an antibody directed against the collagen VI N9-N2 domain (provided by Dr. Rupert Timpl, MPI, Martinsried, Germany), diluted 1:500 in incubation buffer ( $5 \%$ goat serum, $1 \%$ BSA in Tris-buffered saline with $0.05 \%$ Tween-20). The samples were washed with incubation buffer and 
subsequently incubated with secondary alkaline phosphataseconjugated anti-rabbit IgG (Dako, Glostrup, Denmark), diluted $1: 1,000$ in incubation buffer for $2 \mathrm{~h}$ at room temperature. The samples were washed again briefly with incubation buffer. Sections were developed with Vulcan Fast Red chromogen (Biocare Medical, Concord, Calif., USA) and counterstained with Harris hematoxylin (Histolab Products). Sections were visualized using an Eclipse 80i microscope (Nikon, Tokyo, Japan) equipped with a Digital sight DS-Fil camera (Nikon). Statistical evaluations were performed on total 50 different airway profiles on 5 different sections for each individual.

\section{Electron Microscopy and Immunohistochemistry}

For negative staining and transmission electron microscopy, samples were adsorbed to 400-mesh carbon-coated copper grids and stained with $0.75 \%(\mathrm{w} / \mathrm{v})$ uranyl formate as recently described in detail [27]. Samples were examined in a JEOL JEM 1230 transmission electron microscope (JEOL, Peabody, Mass., USA) at 60 $\mathrm{kV}$ accelerating voltage. Images were recorded with a Gatan Multiscan 791 CCD camera using DigitalMicrograph ${ }^{\mathrm{TM}}$ software. For scanning electron microscopy, specimens were fixed overnight at room temperature with $2.5 \%$ glutaraldehyde in cacodylate buffer. They were then washed with cacodylate buffer and dehydrated with an ascending ethanol series from $50 \%(\mathrm{v} / \mathrm{v})$ to absolute ethanol. The specimens were then subjected to critical point drying with carbon dioxide, and absolute ethanol was used as an intermediate solvent. The tissue samples were mounted on aluminum holders, sputtered with $20 \mathrm{~nm}$ palladium/gold and examined in a JEOL JSM-350 scanning electron microscope using a secondary electron detector (Everhart-Tornley detector, JEOL style). Alternatively, tissue samples were coated with 20 -nm carbon prior to immunodetection with a backscattered electron detector (MP64070 - BEIW). Contrast and brightness were adjusted in Adobe Photoshop CS5.

\section{Statistical Analysis}

Data sets were assessed by Student's t test for paired data. Differences were considered statistically significant at $p \leq 0.05$. Significance levels were set at $\mathrm{p} \leq 0.05$ and $\mathrm{p} \leq 0.01$.

\section{Results}

\section{Increased Collagen VI Expression in the Lungs of COPD Patients}

In order to analyze collagen VI mRNA expression in lung tissue, real-time PCR was performed. Tissue samples from control individuals and smokers were compared to COPD patients. Collagen VI mRNA threshold cycle values were normalized to the housekeeping gene GAPDH. We observed that collagen VI mRNA levels were significantly increased in COPD subjects as compared to both healthy subjects and smokers (fig. 1a). No major changes in mRNA levels were observed comparing the control groups of non-smokers and smokers.

Bactericidal Activity of Collagen VI in COPD
Increased Collagen VI Protein Levels in COPD and

Exposure of the Protein in Patches of Epithelial

\section{Damage}

In an attempt to correlate collagen VI gene expression with protein abundance and localization, paraffin sections of lung biopsies from normal and COPD subjects were analyzed by immunohistochemistry and light microscopy. In COPD patients, elevated levels of collagen VI were observed in the extracellular matrix of the airway mucosa. The collagen was particularly abundant in a region $120 \pm 40 \mu \mathrm{m}$ wide and distant to the epithelial basement membrane (fig. 1b, COPD). Interestingly, in patches of epithelial damage, collagen VI in the underlying connective tissue was frequently exposed in the airway lumen (arrow). In contrast, in normal lung tissue, collagen VI exhibited a more ubiquitous distribution throughout the airway mucosa at lower protein expression levels (fig. 1b, control). Similar observations were made in small airways and alveoli (not shown).

\section{Adherence of $M$. catarrhalis to Collagen VI in the}

\section{Airway Mucosa Is Enhanced in COPD}

Based on these results, we first asked whether the increased expression of collagen VI influences the susceptibility of the remodeled airway mucosa to bacterial adherence. Airway biopsies from healthy and COPD subjects were challenged with $M$. catarrhalis for $1 \mathrm{~h}$ at $37^{\circ} \mathrm{C}$, washed extensively and prepared for scanning electron microscopy (fig. 2). Microscopic evaluation showed that there was a significant increase in bacterial adhesion to COPD patient biopsies (fig. 2b) as compared to tissue from control subjects (fig. 2a). Thus, we performed a quantitative evaluation of 30 cellular profiles (fig. 2c). In airways from healthy individuals, bacteria were primarily attached to the ciliated epithelial lining (fig. 2c, healthy). There were only few bacteria binding to intracellular targets and a moderate number adhering to the subepithelial lamina propria. In contrast, in biopsies from COPD patients, $M$. catarrhalis adhesion was greatly enhanced, especially in the upper $\sim 100-\mu \mathrm{m}$ region of the lamina propria, following the tissue distribution of collagen VI described above (fig. 2c, COPD). Frequently bacteria were observed adhering to fibrillar structures in the subepithelial lamina propria (arrows). Similar observations were made in pulmonary fibroblast cell culture and inoculation with M. catarrhalis (fig. 3). Colonies of bacteria frequently adhered to extracellular matrix fibrils secreted by the fibroblasts (fig. 3a, arrows, fig. 3j, k). Scanning electron microscopy and immunolocalization revealed that these fibrils contained collagen VI (fig. 3b, c, arrowheads). 
Similarly, bacteria were observed to adhere to collagen VI-containing fibrils in the lamina propria of lung biopsies (fig. 3f, g, arrowheads).

Inhibition of M. catarrhalis Adherence to the Airway Mucosa and Human Pulmonary Fibroblasts

To further clarify the role of collagen VI in the adherence of $M$. catarrhalis to the airway mucosa, competition studies were performed using polyclonal anti-collagen VI antibodies and soluble collagen VI molecules (fig. 4). Both preincubation of lung biopsies with antibodies and preincubation of bacteria with soluble collagen VI significantly decreased the number of adherent bacteria in the lamina propria (fig. $4 \mathrm{a}-\mathrm{d}$, i). Similar observations were made in parallel experiments with primary pulmonary fibroblasts from healthy individuals and COPD pa-
Fig. 1. Collagen VI is upregulated in COPD airways and exposed in regions of epithelial damage. a RT-PCR of collagen VI expression in lung tissues from healthy individuals, smokers and COPD patients. Relative gene expression was normalized to the housekeeping gene GAPDH and is given as $\Delta \mathrm{C}_{\mathrm{T}}$ value, where higher values correspond to lower gene expression levels. Data sets were assessed by Student's t test for paired data. Differences were considered statistically significant at $p \leq 0.05$. Significance levels were set at $* \mathrm{p} \leq 0.05$ and $* * \mathrm{p} \leq 0.01$. b Immunohistochemistry of airway biopsies obtained from patients with COPD (left panel) and from healthy control individuals (right panel). Collagen VI is widely distributed in the subepithelial lamina propria (control panel). It is overexpressed in COPD, especially in a zone $120 \pm 40 \mu \mathrm{m}$ wide distant to the basement membrane (COPD panel). Regions of epithelial damage and exposure of collagen VI in the underlying connective tissue are frequently observed (arrow). The scale bars represent $500 \mu \mathrm{m}$ (upper panel) and $250 \mu \mathrm{m}$ (lower panel).
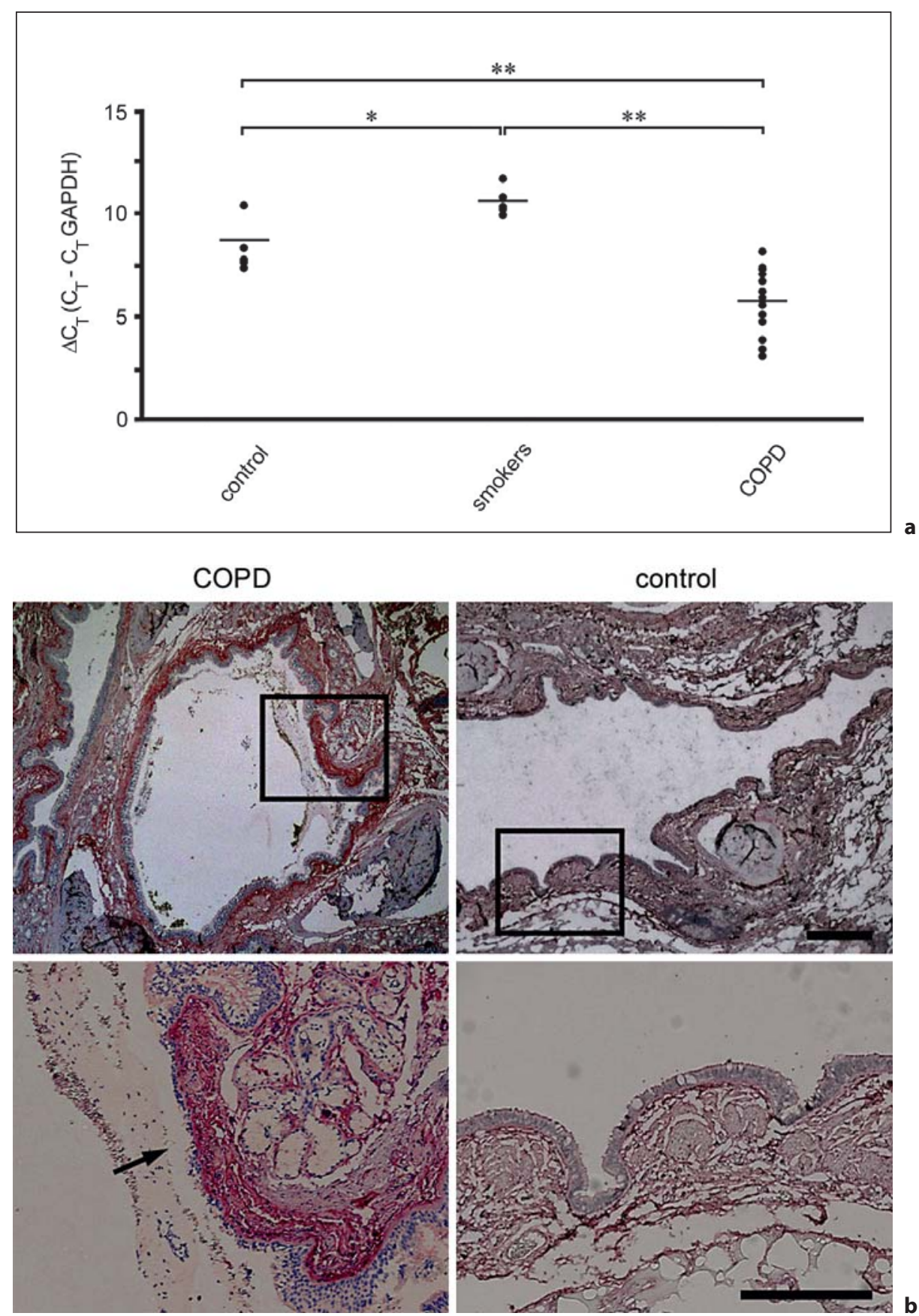
tients (fig. 4e-f, i). There was a 5 -fold increase in bacterial adhesion to airway tissues and fibroblasts from COPD patients, which was decreased to some $40 \%$ by preincubation with the different blocking molecules. The adherence of $M$. catarrhalis bacteria to biopsies or cells was not af- fected by preincubation with nonspecific IgG or human serum albumin (not shown). Taken together, these results reveal the importance of collagen VI as a substrate for $M$. catarrhalis primary adhesion during invasive infection of the bronchial airways in COPD.
Fig. 2. Colonization of $M$. catarrhalis in COPD lung tissues ex vivo. Paraffin sections of lung biopsies from healthy individuals and COPD patients were inoculated with $M$. catarrhalis. In COPD, large amounts of bacteria adhered to the airway mucosa (b) as compared to only moderate binding to tissues from healthy subjects (a). M. catarrhalis is highlighted in green color in $\mathbf{a}$ and $\mathbf{b}$. Frequently bacteria were bound to fibrillar extracellular structures (arrows). The scale bar represents $5 \mu \mathrm{m}$. c Quantification of adherent bacteria found in different compartments of the airway mucosa.
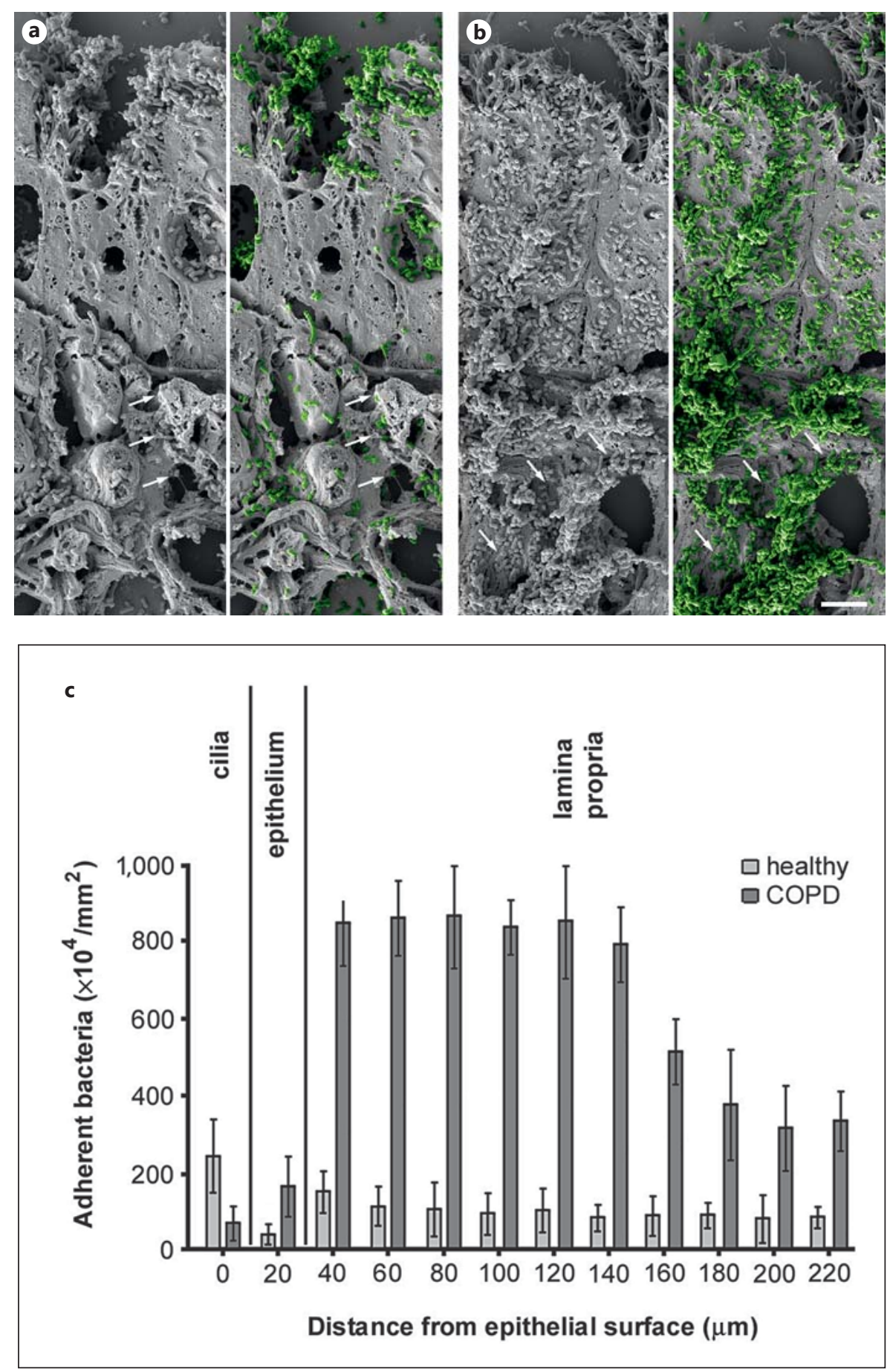

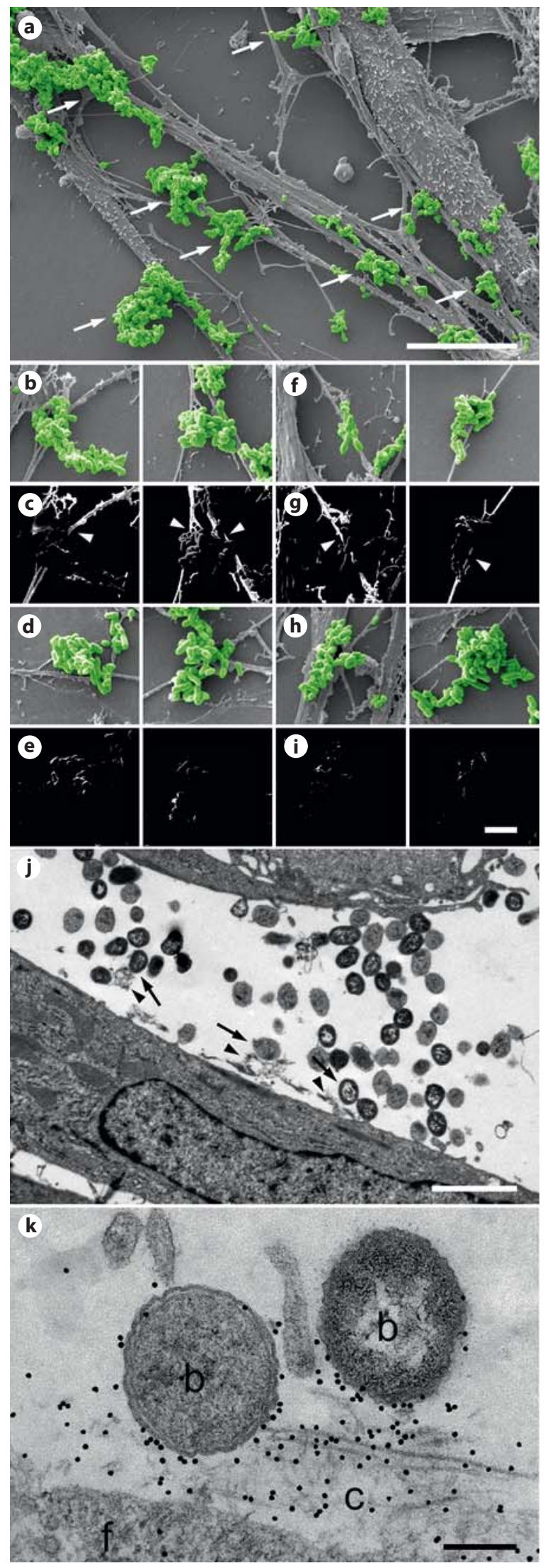

M. catarrhalis Adhere to Collagen VI Microfibrils in vitro, Followed by Membrane Disruption and Killing

Bacteria were incubated for $1 \mathrm{~h}$ at $37^{\circ} \mathrm{C}$ with purified collagen VI microfibrils at physiological $\mathrm{pH}$ and ionic strength ( $\mathrm{pH} 7.4$ and $150 \mathrm{mM} \mathrm{NaCl}$ ). They were compared to a panel of relevant Gram-negative and Grampositive lung pathogens, treated under similar conditions. F. magna isolates from skin were used as a control. Specimens were then examined by negative staining and transmission electron microscopy (fig. 5). Collagen VI microfibrils (arrows) adhered to surface structures of $M$. catarrhalis (fig. 5a). Collagen VI was recruited to the bacterial surfaces of nontypeable $H$. influenzae (fig. 5b), S. pneumoniae (fig. 5c) and Pseudomonas aeruginosa (fig. 5d) in a similar way. In contrast, F. magna showed no affinity for this collagen (fig. 5e). For all pathogens, collagen VI binding resulted in membrane destabilization, exudation of cytoplasmic content (fig. $5 f-i$, asterisks) and killing (fig. 6a). Electron-microscopic inspection of the events underlying the killing process revealed extensive initial membrane vesicle blebbing, followed by large-scale membrane disruption (fig. 6b-g). F. magna bacteria were not affected and retained their morphological integrity (fig. 5j, 6a).

\section{M. catarrhalis Interact Specifically with Collagen VI through the Collagen VI $\alpha_{2}$-Chain}

Intact collagen VI microfibrils and a panel of recombinant fragments thereof (fig. 7a) were radiolabeled with ${ }^{125} \mathrm{I}$ and added to $M$. catarrhalis suspensions at physiological conditions. In control experiments, ${ }^{125}$ I-labeled collagen

Fig. 3. M. catarrhalis bind to collagen VI-containing extracellular matrix fibrils. Human lung fibroblasts from patients with severe COPD (GOLD stage IV; $\mathbf{a}, \mathbf{c}$ ) were allowed to deposit a dense fibrillar matrix. The specimens were incubated with $M$. catarrhalis and prepared for immunolocalization and scanning electron microscopy. The bacteria were frequently observed to adhere to extracellular collagen fibrils (arrows). These fibrils contained collagen VI as shown by immunodetection with collagen VI antibodies (b, c, arrowheads). Similar observations were made with collagen fibrils in airway biopsies described in figure $2(\mathbf{f}, \mathbf{g})$. Control specimens treated with secondary gold conjugate alone did not exhibit any fibrillar staining (d, e, fibroblasts; $\mathbf{h}$, i, airway biopsies). The scale bars represent $10 \mu \mathrm{m}(\mathbf{a})$ and $2.5 \mu \mathrm{m}(\mathbf{b}-\mathbf{i})$. j, k Ultrathin sections of fibroblasts incubated with $M$. catarrhalis bacteria (arrows) adhering to the extracellular collagen matrix (arrowheads). k Bacteria are frequently colocalized with collagen VI in the extracellular collagen matrix as visualized by collagen VI antibodies conjugated with 5 -nm colloidal gold. $\mathrm{f}=$ Fibroblast; $\mathrm{b}=$ bacteria; $\mathrm{c}=$ extracellular collagen matrix. The scale bars represent $1 \mu \mathrm{m}$. 
VI microfibrils were added to PBS without bacteria. After incubation for $1 \mathrm{~h}$ at $37^{\circ} \mathrm{C}$, followed by centrifugation and washing steps, full-length collagen and the collagen VI $\alpha_{2}$ chain were found in the bacterial pellets (fig. 7b). Similar observations were made by transmission electron microscopy (fig. 7c). Collagen VI fragments were labeled with 5-nm colloidal gold and incubated with $M$. catarrhalis bacteria. Negative staining revealed that the recombinant collagen VI $\alpha_{2}$-chain bound specifically to the bacterial surface (arrowheads) whereas the other collagen fragments exhibited a random background distribution.

\section{Discussion}

Recently, we described collagen VI as a multifunctional extracellular matrix molecule with adhesive and antimicrobial properties against Gram-positive pathogens in vitro [22]. In this study, we set out to further dissect the molecular mechanisms regulating the role of this intriguing molecule in innate host defense. We elucidate potential in vivo functions as an adhesive substrate and antibacterial agent for Gram-negative respiratory microorganisms. M. catarrhalis is used as a model system and in some experiments compared to other relevant pulmonary pathogens such as nontypeable $H$. influenzae, S. pneumoniae and $P$. aeruginosa. In particular, the susceptibility of the airway mucosa to M. catarrhalis infection in COPD is assessed. We identified the collagen VI $\alpha_{2}$-chain as the primary adhesion target, a hitherto unknown feature, giving this collagen its adhesive properties. Upon collagen VI recruitment on the bacterial surface, $M$. catarrhalis and other pathogens are rapidly eliminated by membrane disruption. These findings highlight the many faces of collagen VI in bacterial host invasion and in innate host defense transmitted by the extracellular matrix.

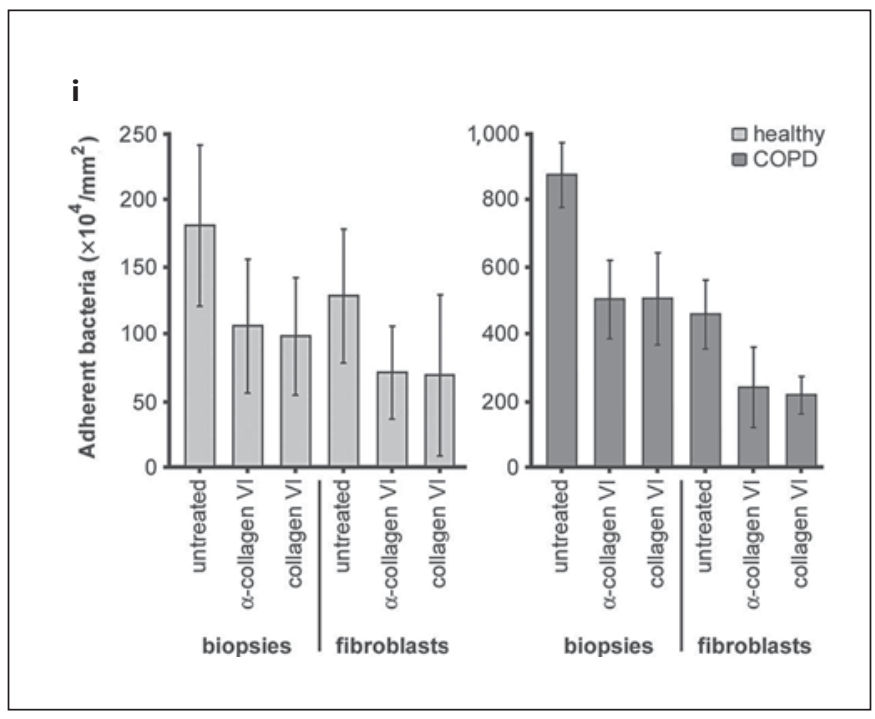

Fig. 4. Blocking of $M$. catarrhalis adherence to the airway mucosa and human pulmonary fibroblasts. Scanning electron micrographs of paraffin sections of airway biopsies (a-d) or pulmonary fibroblasts (e-h) from healthy individuals (a, b, biopsies; e, f, fibroblasts) and COPD patients (c, $\mathbf{d}$, biopsies; $\mathbf{g}, \mathbf{h}$, fibroblasts) inoculated with $M$. catarrhalis bacteria in the absence $(\mathbf{a}, \mathbf{c}, \mathbf{e}, \mathbf{g})$ or presence $(\mathbf{b}, \mathbf{d}, \mathbf{f}, \mathbf{h})$ of $\alpha$-collagen VI antibodies. The scale bars represent $5 \mu \mathrm{m}$. i Quantitative evaluation of adherent bacteria.
Bactericidal Activity of Collagen VI in COPD
J Innate Immun 2015;7:506-517 DOI: $10.1159 / 000381213$ 
Collagen VI is a member of an extracellular matrix protein family which is ubiquitous in the body, making up $25-35 \%$ of the total protein content. Collagens are found in skin, lungs, tendons (type I collagen), basement membranes (type IV collagen) and joints (type II collagen). Collagen VI has an interstitial distribution, often located distant to basement membranes and associated with collagen I. In addition, it has recently been proposed as a basement membrane component [16]. Studies on pathogen adherence to collagens are mainly focused on the classical fibrillar collagens type I, III and V, or the basement membrane collagen type IV [for references, see 10]. However, only a few pathogens have been described to bind collagen directly, and the majority of them use prebound fibronectin as a bridging molecule [8]. Here, we describe a novel function for collagen VI as a substrate for direct adherence of $M$. catarrhalis. Similar observations have been made for a variety of Gram-positive and Gram-negative pathogens [Abdillahi et al., in preparation; Nordin et al., in preparation], introducing this molecule as a broad-spectrum antimicrobial agent. It thus appears that collagen VI renders the host extracellular matrix susceptible to pathogen attachment during distinct stages of infection. In a following series of events, this collagen enables the host to clear the infection site from invading pathogens by rapid killing.

Invasion of the airway mucosa requires intricate strategies by pathogens, trying to evade the various types of host response. Bacterial anchorage on host targets is an initial crucial step in the process of adhesion, colonization and establishment of a biofilm, and the subsequent development into a clinically relevant invasive infection. In response to microbial encounter, the host has evolved multiple innate and adaptive defense mechanisms for efficient pathogen eradication and tissue repair while limiting collateral tissue damage. Therefore, one of the most important strategies to combat devastating pulmonary infections is to understand the molecular mechanisms of

Fig. 5. Collagen VI microfibrils adhere to M. catarrhalis and other pulmonary pathogens, followed by killing. Collagen VI microfibrils were purified from bovine cornea and allowed to react with $M$. catarrhalis (a), nontypeable $H$. influenzae (b), S. pneumoniae (c) and $P$. aeruginosa (d). Negative staining and transmission electron microscopy visualizes primary adhesion of collagen VI networks (arrows) to the bacterial surfaces (a-d), followed by killing within $1 \mathrm{~h}(\mathbf{f}-\mathbf{i})$. Asterisks denote areas of membrane destabilization and exudation of intracellular content $(\mathbf{f}-\mathbf{i})$. F. magna was used as a control and did neither recruit collagen VI (e) nor exhibit killing (j). The scale bars represent $100 \mathrm{~nm}(\mathbf{a}-\mathbf{e})$ and $1 \mu \mathrm{m}$ $(\mathbf{f}-\mathbf{j})$. early phases of pathogen adhesion and innate host responses.

Thus, in initial experimental setups, collagen VI expression in the airways of healthy and COPD subjects was investigated. By a combination of RT-PCR and immuno-
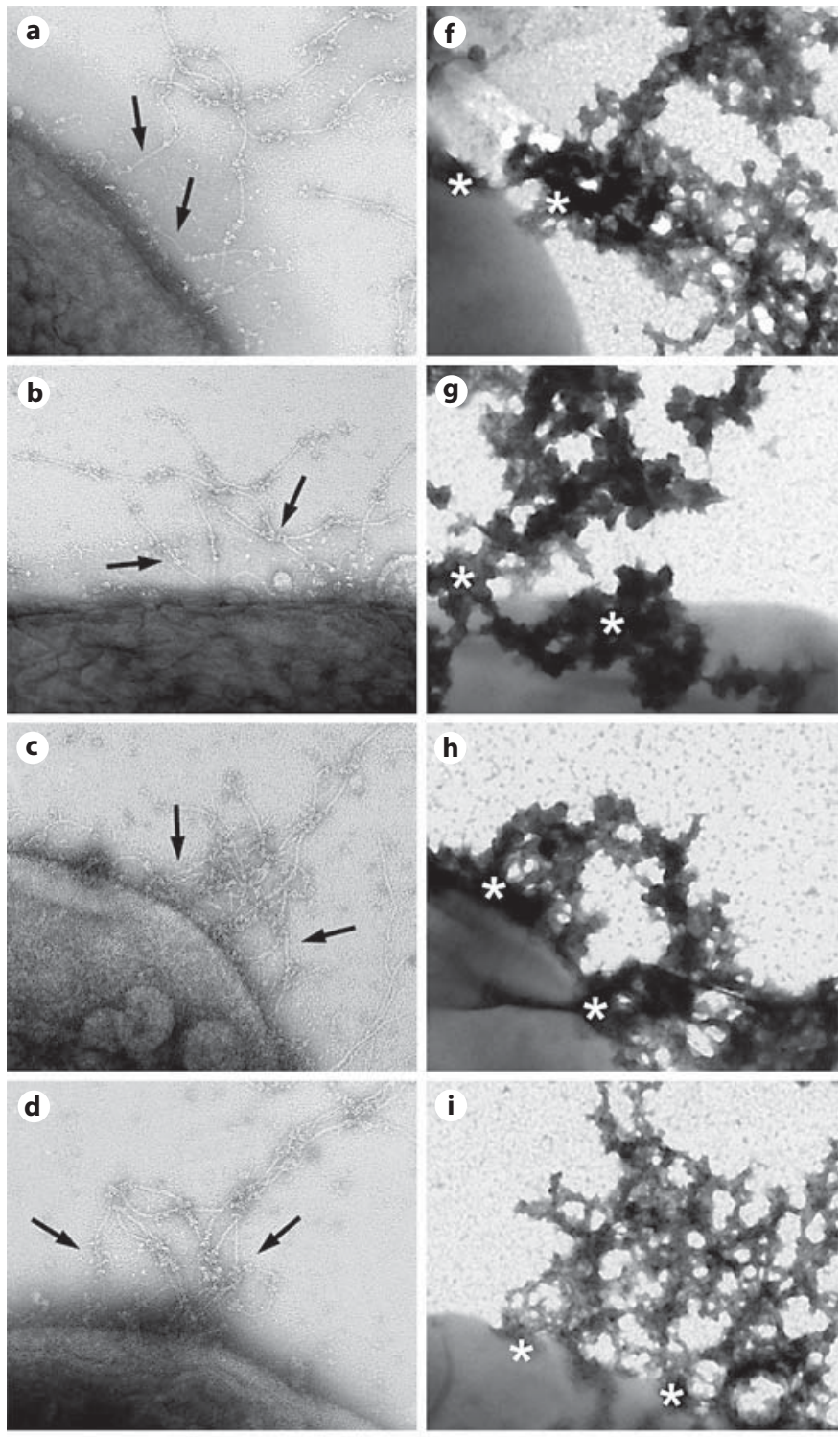

e

j

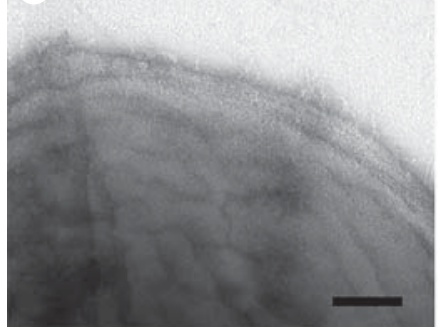


histochemistry, we found a broad distribution of the protein in the lamina propria. In contrast, the collagen was significantly upregulated in fibrotic airways, where the highest expression levels were found in a 100 - to $200-\mu \mathrm{m}$ wide region distant to the subepithelial basement membrane. As a consequence, $M$. catarrhalis adherence was considerably enhanced in COPD patients. The bacteria strongly bound to collagen VI-containing fibrillar networks and followed the collagen VI expression pattern through the lamina propria. Pretreatment of specimens with soluble collagen VI protein or anticollagen VI antibodies reduced the binding capability of $M$. catarrhalis. Similar observations were made with pulmonary fibroblasts in cell culture. These data suggest significant contributions of collagen VI in mediating primary pathogen adhesion in the pulmonary system.

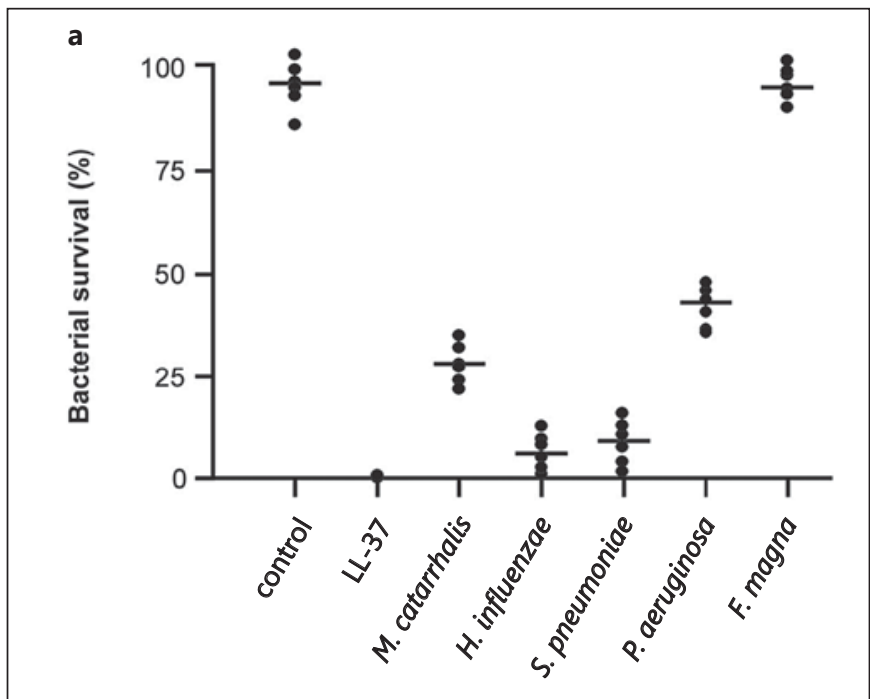

Fig. 6. Collagen VI microfibrils induce killing of pulmonary pathogens by membrane destabilization. Bacteria were incubated with $1 \mathrm{mM}$ collagen VI for $2 \mathrm{~h}$ at $37^{\circ} \mathrm{C}$. a Numbers of bacterial colonies after incubation with collagen VI were determined in viable count assays. Incubation in buffer alone (control) or LL-37 served as negative and positive controls, respectively. The dots represent individual experiments with the respective strains. F. magna were not killed and served as a negative control. Values are expressed as percent bacterial survival. $\mathbf{b}-\mathbf{g}$ Negative staining and transmission electron microscopy of $M$. catarrhalis $(\mathbf{b}, \mathbf{d}, \mathbf{f})$ and $P$. aeruginosa (c, e, g) bacteria. Upon incubation with collagen VI, large-scale membrane disruption results in destruction of the pathogens (d, e) as compared to controls incubated with TBS alone (b, c). When the bacteria are beginning to die, blebbing of membrane vesicles (arrowheads) is frequently observed $(\mathbf{f}, \mathbf{g})$. Similar observations were made for the killing of $H$. influenzae and S. pneumoniae (not shown). The scale bars represent $1 \mu \mathrm{m}$ (b-e) and $0.5 \mu \mathrm{m}(\mathbf{f}, \mathbf{g})$.
Structural abnormalities caused by airway remodeling are currently known to affect not only individuals with asthma, but also patients with COPD $[28,29]$. They include epithelial desquamation and eventually epithelial denudation, followed by exposure of the subepithelial basement membrane and the underlying lamina propria. Postviral infection injury is likely to have the same effect by breaching the airway mucosa integrity. In patches of epithelial lesions, a variety of extracellular matrix targets become exposed and may provide entry ports for invasive microorganisms. In this environment, elevated levels of collagen VI networks with both adhesive and antimicrobial properties may be beneficial for containment and rapid elimination of pathogen intruders already at the basement membrane barrier. Indeed, the results presented in this work show that different pulmonary pathogens are rapidly killed by collagen VI microfibrils, sug-

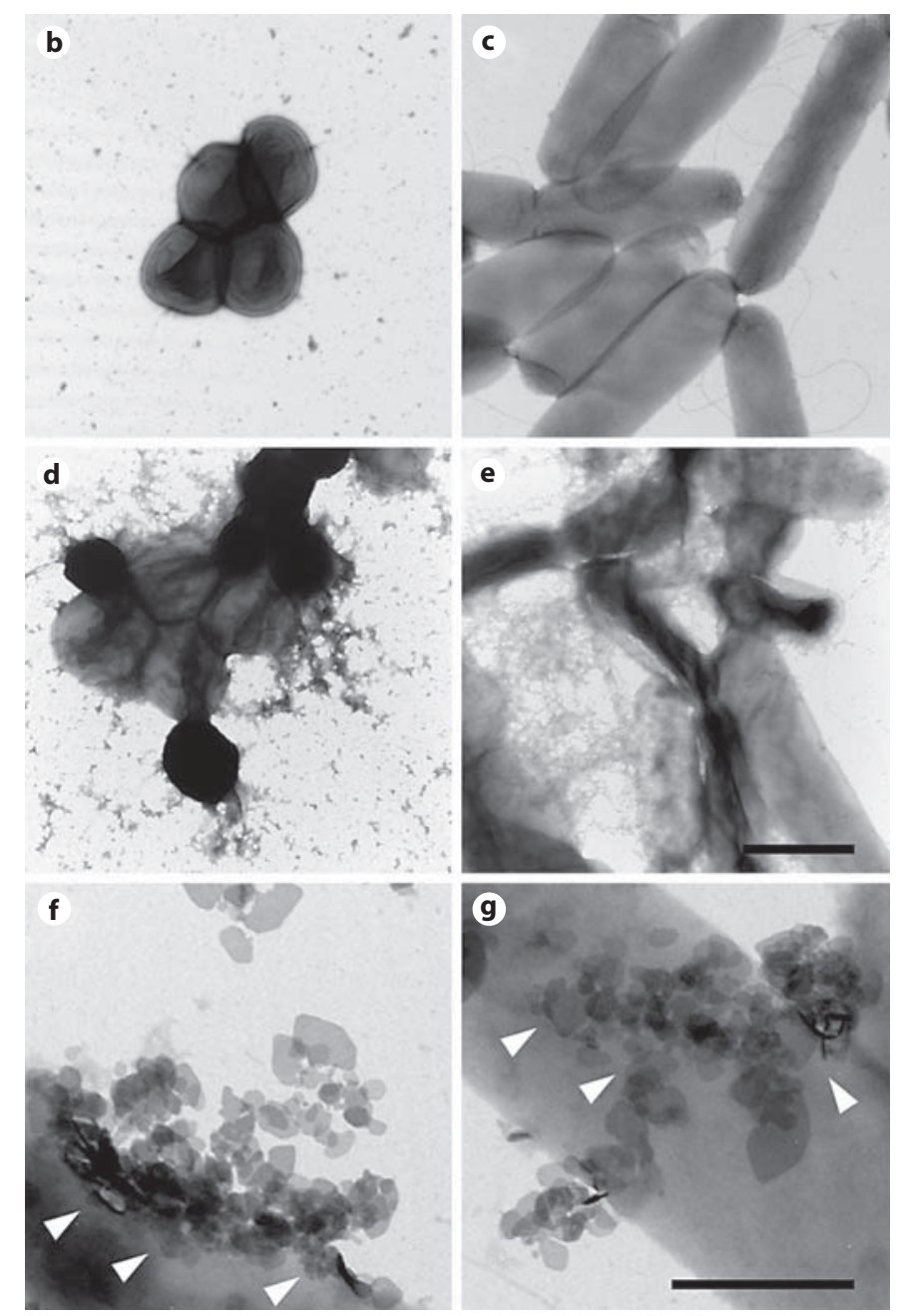



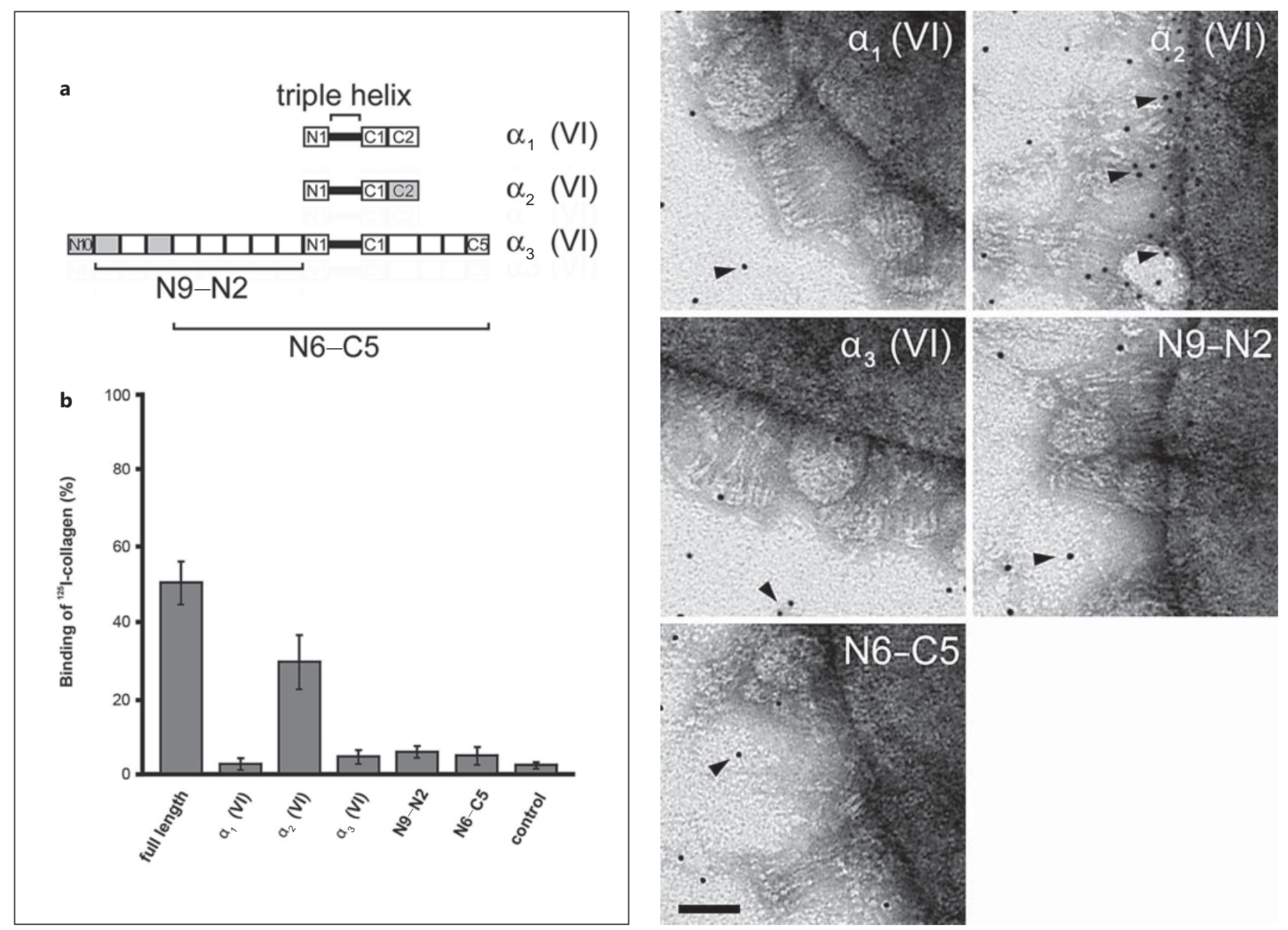

Fig. 7. The collagen VI $\alpha_{2}$-chain interacts specifically with $M$. catarrhalis. Recombinant collagen VI $\alpha$-chains $\left(\alpha_{1}\right.$, $\alpha_{2}$ and $\alpha_{3}$ ) and $\alpha_{3}$-chain fragments (N9-N2 and N6-C5; schematic overview in a) were labeled with ${ }^{125} \mathrm{I}(\mathbf{b})$ or colloidal gold (c) and allowed to interact with $M$. catarrhalis. Only full-length collagen VI and the collagen VI $\alpha_{2}$-chain were specifically associated with bacteria (b, c, arrowheads). The scale bar represents $50 \mathrm{~nm}$.

gesting an important role of this molecule in host defense in vivo. Similarly, host defense properties of the basement membrane protein laminin have recently been described [30, 31], highlighting the emerging position of connective tissue components in the field of innate immunity. However, the exact molecular mechanisms underlying such properties of collagen VI in vivo are still elusive and beyond the scope of this work. It remains an exciting future challenge to investigate how in vivo degradation mechanisms generate bioactive peptides from collagen VI.

Taken together, according to this mechanism, adherence to collagen VI scaffolds may support pathogen ag- gregation, colonization and host invasion, causing infections of deeper tissue compartments and contributing to COPD exacerbations. On the other hand, pathogen recruitment on collagen VI may be a means for the host to prevent pathogen spreading by containment and rapid elimination. The in vivo relevance of collagen type VI for primary adhesion as well as the characterization of the microbial surface adhesins is currently investigated in our group in more detail. Our findings contribute to an increasing body of knowledge of the carefully orchestrated balance between host invasion and host defense in bacterial infections. 


\section{Acknowledgments}

The authors gratefully acknowledge Rita Wallén, Cell and Organism Biology, Lund University, and the Core Facility for Integrated Microscopy, Panum Institute, University of Copenhagen, for help with electron microscopy. This work was supported by grants from the Swedish Research Council (project No. 7480), the Swedish Foundation for Strategic Research, the Foundations of Crafoord, Johan and Greta Kock, Alfred Österlund, King Gustav V Memorial Fund, and the Medical Faculty at Lund University.

\section{References}

1 Karalus R, Campagnari A: Moraxella catarrhalis: a review of an important human mucosal pathogen. Microbes Infect 2000;2:547559.

2 Verduin CM, Hol C, Fleer A, van Dijk H, van Belkum A: Moraxella catarrhalis: from emerging to established pathogen. Clin $\mathrm{Mi}$ crobiol Rev 2002;15:125-144.

-3 Aebi C: Moraxella catarrhalis - pathogen or commensal? Adv Exp Med Biol 2011;697: 107-116.

4 Stenfors LE, Raisanen S: Interaction between Streptococcus pneumoniae and Branhamella catarrhalis obtained from double-colonized, healthy nasopharynx and double-infected, diseased middle-ear cavity. Scand J Infect Dis 1989;21:397-401.

-5 Murphy TF, Parameswaran GI: Moraxella catarrhalis, a human respiratory tract pathogen. Clin Infect Dis 2009;49:124-131.

-6 Parameswaran GI, Wrona CT, Murphy TF, Sethi S: Moraxella catarrhalis acquisition, airway inflammation and protease-antiprotease balance in chronic obstructive pulmonary disease. MBC Infect Dis 2009;9:178.

7 Patti JM, Allen BL, McGavin MJ, Höök M MSCRAMM-mediated adherence of microorganisms to host tissues. Annu Rev Microbiol 1994;48:585-617.

$\checkmark 8$ Dinkla K, Rohde M, Jansen WT, Carapetis JR, Chhatwal GS, Talay SR: Streptococcus pyogenes recruits collagen via surface-bound fibronectin: a novel colonization and immune evasion mechanism. Mol Microbiol 2003;47 861-869.

-9 Nitsche DP, Johansson HM, Frick IM, Mörgelin M: Streptococcal protein FOG, a novel matrix adhesin interacting with collagen $\mathrm{I}$ in vivo. J Biol Chem 2006;281:1670-1679.

10 Dinkla K, Talay SR, Mörgelin M, Graham RM, Rohde M, Nitsche-Schmitz P, Chhatwal GS: Crucial role of the CB3-region of collagen IV in PARF-induced acute rheumatic fever. PLoS One 2009; 4:e4666.

11 Hallström T, Singh B, Resman F, Blom AM, Mörgelin M, Riesbeck K: Haemophilus influenzae protein $\mathrm{E}$ binds to the extracellular matrix by concurrently interacting with laminin and vitronectin. J Infect Dis 2011;204:10651074.
12 Jalalvand F, Su YC, Mörgelin M, Brant M, Hallgren O, Westergren-Thorsson G, Singh B, Riesbeck K: Haemophilus influenzae protein $\mathrm{F}$ mediates binding to laminin and human pulmonary epithelial cells. J Infect Dis 2013;207:803-813.

13 Singh B, Fleury C, Jalalvand F, Riesbeck K: Human pathogens utilize host extracellular matrix proteins laminin and collagen for adhesion and invasion of the host. FEMS Microbiol Rev 2012;36:1122-1180.

14 Hessle H, Engvall E: Type VI collagen. Studies on its localization, structure, and biosynthetic form with monoclonal antibodies. J Biol Chem 1984;259:3955-3961.

15 Bruns RR, Press W, Engvall E, Timpl R, Gross J: Type VI collagen in extracellular, 100-nm periodic filaments and fibrils: identification by immunoelectron microscopy. J Cell Biol 1986;103:393-404.

16 Groulx JF, Gagné D, Benoit YD, Martel D, Basora N, Beaulieu JF: Collagen VI is a basement membrane component that regulates epithelial cell-fibronectin interactions. Matrix Biol 2011;3:195-206.

17 Colombatti A, Bonaldo P, Ainger K, Bressan GM, Volpin D: Biosynthesis of chick type VI collagen. I. Intracellular assembly and molecular structure. J Biol Chem 1987;262:1445414460.

18 Gara SK, Grumati P, Urciuolo A, Bonaldo P, Kobbe B, Koch M, Paulsson M, Wagener R: Three novel collagen VI chains with homology to the alpha3 chain. J Biol Chem 2008;283: 10658-10670.

19 Fitzgerald J, Rich C, Zhou FH, Hansen U: Three novel collagen VI chains, alpha $4 \mathrm{VI}$, alpha 5 VI and alpha 6 VI. J Biol Chem 2008; 283:20170-20180.

20 Malmström J, Tufvesson E, Löfdahl CG, Hansson L, Marko-Varga G, WestergrenThorsson G: Activation of platelet-derived growth factor pathway in human asthmatic pulmonary-derived mesenchymal cells. Electrophoresis 2003;24:276-285.

21 Bober M, Enochsson C, Collin M, Mörgelin $\mathrm{M}$ : Collagen VI is a subepithelial adhesive target for human respiratory tract pathogens. J Innate Immun 2010;2:160-166.

22 Abdillahi SM, Balvanović S, Baumgarten M, Mörgelin M: Collagen VI encodes antimicrobial activity: novel innate host defense properties of the extracellular matrix. J Innate Immun 2012;4:371-376.
23 Livak KJ, Schmittgen TD: Analysis of relative gene expression data using real-time quantitative PCR and the $2^{-\Delta \Delta C t}$ method. Methods 2001;25:402-408.

24 Spissinger T, Engel J: Type VI collagen beaded microfibrils from bovine cornea depolymerize at acidic $\mathrm{pH}$, and depolymerization and polymerization are not influenced by hyaluronan. Matrix Biol 1995;14:499-505.

25 Timpl R, Chu M-L: Microfibrillar collagen type VI; in Yurchenko PD, Birk D, Mecham RP (eds): Extracellullar Matrix Assembly and Structure. Orlando, Academic Press, 1994, pp 207-242.

26 Baschong W, Wrigley NG: Small colloidal gold conjugated to Fab fragments or to immunoglobulin $\mathrm{G}$ as high-resolution labels for electron microscopy: a technical overview. J Electron Microsc Tech 1990;14:313-323.

27 Oehmcke S, Mörgelin M, Herwald H: Activation of the human contact system on neutrophil extracellular traps. J Innate Immun 2009; 1:225-230.

28 Kosciuch J, Krenke R, Gorska K, Baran W, Kujawa M, Hildebrand K, Chazan R: Comparison of airway wall remodeling in asthma and COPD: biopsy findings. Respir Care 2012;57:557-564.

-29 Ojo O, Lagan AL, Rajendran V, Spanjer A, Chen L, Singh Sohal S, Heijink I, Jones R, Maarsingh $\mathrm{H}$, Hackett TL: Pathological changes in the COPD lung mesenchyme novel lessons learned from in vitro and in vivo studies. Pulm Pharmacol Ther 2014;29:121128 .

30 Senyürek I, Klein G, Kalbacher H, Deeg M, Schittek B. Peptides derived from the human laminin alpha4 and alpha5 chains exhibit antimicrobial activity. Peptides 2010;31:14681472.

31 Senyürek I, Kempf WE, Klein G, Maurer A, Kalbacher H, Schäfer L, Wanke I, Christ C, Stevanovic S, Schaller M, Rousselle P, Garbe C, Biedermann T, Schittek B: Processing of laminin a chains generates peptides involved in wound healing and host defense. J Innate Immun 2014;6:467-484. 